\title{
Unraveling complex magnetism in two-dimensional $\mathrm{FeS}_{2}$
}

\author{
Duo Wang $\odot,{ }^{*}$ Xin Chen $\odot,{ }^{\dagger}$ and Biplab Sanyal $\oplus^{\ddagger}$ \\ Department of Physics and Astronomy, Uppsala University, 75120 Uppsala, Sweden
}

(Received 10 June 2021; revised 17 November 2021; accepted 19 November 2021; published 9 December 2021)

\begin{abstract}
Atomically thin two-dimensional (2D) magnets have given rise to emergent phenomena due to magnetic exchange and spin-orbit coupling showing great promise for realizing ultrathin device structures. In this paper, we critically examine the magnetic properties of $2 \mathrm{D} \mathrm{FeS}_{2}$, a non van der Waals magnet, which has been recently claimed to exhibit room-temperature ferromagnetism in the (111) orientation. Our $a b$ initio study based on collinear density functional theory has revealed the ground state as an antiferromagnetic one with an ordering temperature of around $100 \mathrm{~K}$ along with a signature of spin-phonon coupling, which may trigger a ferromagnetic coupling via strain. Moreover, our calculations based on spin spirals indicate the possibility of noncollinear magnetic structures, which is also supported by Monte Carlo simulations based on ab initio magnetic exchange parameters. This opens up an excellent possibility to manipulate magnetic structures by the application of directional strain.
\end{abstract}

DOI: 10.1103/PhysRevB.104.245410

\section{INTRODUCTION}

Magnetism in two dimensions (2D) has long been at the heart of numerous theoretical, experimental, and technological advances as thi research area has been proven to be a fertile ground for emergent magnetic phenomena, such as the study of topology [1-3], multiferroicity [4-7], proximity effects in heterostructures [8], etc. Theoretically, long-range magnetic order is prohibited in the $2 \mathrm{D}$ isotropic-Heisenberg model at a finite temperature by the Mermin-Wagner theorem. However, symmetry breaking, such as the occurrence of magnetic anisotropy, removes this restriction. The discovery of $\mathrm{CrI}_{3}$, a 2D material that retains ferromagnetic ordering in a monolayer [9], has opened a new era of tunable magnetism in the ultrathin scale. A number of 2D magnets have emerged after 2017, such as $\mathrm{Cr}_{2} \mathrm{Ge}_{2} \mathrm{Te}_{6}$ [10], $\mathrm{Fe}_{3} \mathrm{GeTe}_{2}$ [11], $\mathrm{Cr} X_{3}(X=\mathrm{Cl}$, Br) [12,13], $\mathrm{MnSe}_{2}$ [14], $M \mathrm{PS}_{3}(M=\mathrm{Ni}, \mathrm{Mn}, \mathrm{Co})$ [15-17], $\mathrm{CrTe}_{2}$ [18], and $\mathrm{Cr}_{2} \mathrm{Te}_{3}$ [19]. The weak van der Waals bonding between layers in bulk 3D magnetic systems offers a unique advantage to realize 2D or quasi-2D forms of these systems. However, the challenge is to have a magnetic long-range order and thermal stability at room temperature and above.

Bulk $\mathrm{FeS}_{2}$ is a paramagnetic material with a band gap around $0.9 \mathrm{eV}$, which makes it a promising candidate for applications in the field of photovoltaic and photoelectron chemical cells [20-22]. $\mathrm{FeS}_{2}$ is similar to $\mathrm{MnSe}_{2}$ [14], which

\footnotetext{
*Duo.Wang@physics.uu.se

$\dagger$ cchenxinn@gmail.com

†Biplab.Sanyal@physics.uu.se
}

has been demonstrated to have high-temperature ferromagnetism down to monolayers whereas their bulk counterparts are low-temperature antiferromagnetic materials. In the past, the dynamical stability, and electronic and magnetic properties of $2 \mathrm{D} \mathrm{FeS}$ with different surface orientations have been investigated theoretically [23-27]. Very recently, Anand et al. have reported the isolation of $2 \mathrm{D} \mathrm{FeS}_{2}$, and more importantly, they found that it is possible to observe long-range ferromagnetic (FM) order even up to room temperature [28,29]. Their density functional theory (DFT) simulations show that the $\mathrm{FeS}_{2}$ (111) surface is the one that has the lowest formation energy and at the same time has a FM configuration as the ground state. However, the occurrence of room-temperature ferromagnetism is quite intriguing and calls for a critical analysis.

In this paper, our aim is to investigate the properties of $\mathrm{FeS}_{2}$ (111) monolayer in detail. First, we found that a structure with an antiferromagnetic (AFM) configuration is more stable than the FM structure, the energy difference being quite small and sensitive to the crystal structure, which indicates that there is a substantial spin-lattice coupling. Furthermore, we studied the interatomic magnetic exchange parameters and ordering temperatures for AFM and FM structural phases. The results show that the ordering temperatures are 102 and $32 \mathrm{~K}$ for crystal structures with AFM and FM coupling, respectively. Moreover, spin-spiral calculations utilizing the generalized Bloch theorem indicate that the magnetic ground state of the (111) surface is actually noncollinear, which is also consistent with our results obtained from Monte Carlo simulations where the canting of magnetic moments deviating from the collinear magnetic configuration is observed.

\section{COMPUTATIONAL METHODS}

Our computational approach is based on density functional theory [30] using the plane-wave basis set and projector augmented-wave (PAW) method $[31,32]$ implemented in the 
Vienna ab initio simulation package (VASP)[33]. The planewave energy cutoff was set to $600 \mathrm{eV}$. A $11 \times 11 \times 1 k$ mesh was used for integration in the Brillouin zone, and the forces on atoms were converged to $0.001 \mathrm{eV} / \AA$. The optimized structures were used as input for the full-potential linearized muffin-tin orbital (FP-LMTO) code RSPT [34] to calculate the magnetic anisotropy energy (MAE) and interatomic magnetic exchange parameters. The calculations of interatomic exchange parameters were based on magnetic force theorem (MFT) [35], which maps the magnetic excitations onto the Heisenberg Hamiltonian,

$$
\hat{H}=-\sum_{i \neq j} J_{i j} \vec{e}_{i} \cdot \vec{e}_{j}
$$

where $J_{i j}$ is the interatomic exchange interaction between the two magnetic moments at sites $i$ and $j$, and $e$ is the unit vector along the magnetization direction at site $i$ (or $j$ ). The orbitally resolved interatomic exchange parameter has the following expression,

$$
J_{i j}^{m_{1} m_{2}}=\frac{T}{4} \sum_{-\infty}^{\infty}\left[\tilde{\hat{\Delta}}_{i, m_{1}} \cdot G_{i j, m_{1} m_{2}}^{\uparrow} \cdot \tilde{\hat{\Delta}}_{j, m_{2}} \cdot G_{j i, m_{2} m_{1}}^{\downarrow}\right],
$$

in which $\hat{G}_{i j}$ is the real-space intersite Green's function, and $\hat{\Delta}_{i}$ is the exchange splitting on site $i$. Theoretically, it is possible to obtain an orbitally decomposed exchange parameter for the system in any basis since both $\hat{G}_{i j}$ and $\hat{\Delta}_{i}$ are orbitally dependent. However, the above method has not been implemented yet in the code and therefore we can only roughly get " $t_{2 g}$-like" and " $e_{g}$-like" states by diagonalizing the intersite $J_{i j}$ matrix. The $k$ mesh in the Brillouin zone was performed using $31 \times 31 \times 1$ points. To describe the exchange-correlation effects, we used the generalized gradient approximation (GGA) [36] augmented by the Hubbard- $U$ correction $\left(\mathrm{GGA}+U_{\text {eff }}\right)$ [37]. The Coulomb $U_{\text {eff }}(U-J)$ value we used for Fe- $d$ electrons is $2 \mathrm{eV}$, which has been proven to be a good value for $2 \mathrm{D} \mathrm{Fe}$ structures $[28,38]$. In our simulations, we took $\mathrm{Fe}$ $3 s 3 p 3 d 4 s$ and $\mathrm{S} 3 s 3 p$ electrons as valence electrons. Finally, we used the extracted $J_{i j}$ and MAE to calculate the magnetic ordering temperature by means of classical Monte Carlo (MC) simulations for the solution of the Heisenberg Hamiltonian, and also adiabatic magnon spectra as implemented in the Uppsala atomistic spin dynamics (UPPASD) [39] code. For this purpose, a $70 \times 70 \times 1$ (19 600 atoms) cell was considered. The transition state barriers are obtained by using the climbing image nudged elastic band (cNEB) method [40].

\section{RESULTS}

The $\mathrm{FeS}_{2}$ (111) surface was considered due to its lowest formation energy and dynamical stability [27,28]. The crystal structure belongs to the family of layered transition metal dichalcogenides, in which two $\mathrm{S}$ layers sandwich the Fe layer. There are six nearest-neighbor Fe atoms around each $\mathrm{Fe}$ atom, forming a hexagonal close-packed structure. Each Fe atom (nominal charge state as $\mathrm{Fe}^{4+}$ ) with six nearest-neighbor $\mathrm{S}$ atoms form an octahedral structure offering a crystal field splitting of $d$ states into $t_{2 g}$ (three electrons) and $e_{g}$ (one electron) states. Our accurate calculations of total energies (confirmed by both plane-wave PAW and FP-LMTO methods) indicate that the $\mathrm{FeS}_{2}$ (111) surface with an AFM config- (a)
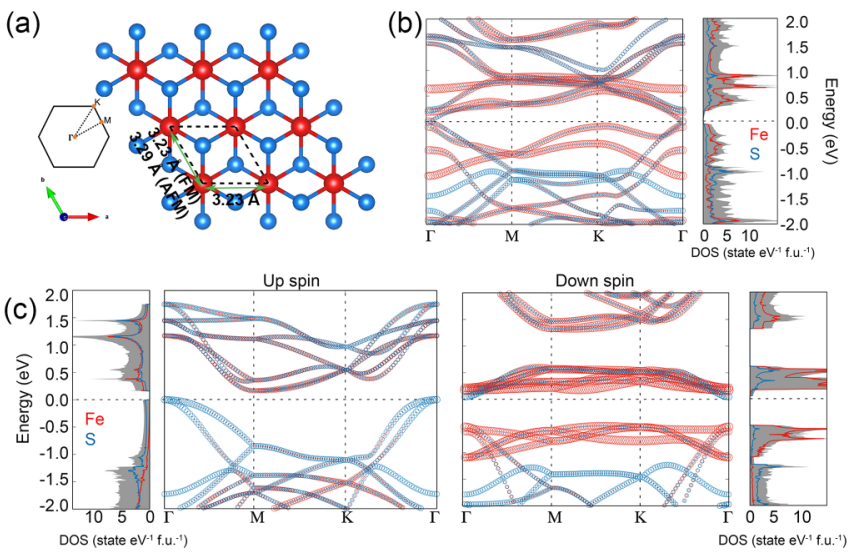

FIG. 1. (a) The top view of (111) surface. Fe and S atoms are indicated as blue and red balls, respectively. The unit cell is shown in the dashed line, and $\mathrm{Fe}-\mathrm{Fe}$ distances are marked with green arrows. The first Brillioun zone with high symmetry points is shown. Density of states (DOS) and atom-projected band structure for up-spin and down-spin electrons of the (b) AFM structure and (c) FM structure are shown. Total DOS (shaded regions) and partial DOS of Fe (red curves) and $\mathrm{S}$ (blue curves) are shown. In the projected band structures, the size of the circles is proportional to the weight of the atomic contribution. The Fermi level is set to $0 \mathrm{eV}$.

uration has lower energy compared to the FM state, which is in contrast to some previous studies [27,29]. However, the energy difference between these two phases is relatively small $(7.2 \mathrm{meV} /$ atom $)$ and is sensitive to the crystal structure. This result, on the other hand, explains the observation in the experiment [29] very well. Due to the coexisting FM and AFM phases, the opening of the $M-H$ hysteresis loop does not reach saturation under high magnetic fields. The top views of these two structures are shown in Fig. 1(a). The FM structure is symmetrical with all the next-nearestneighbor Fe-Fe distances being exactly the same, whereas for the structure with AFM Fe-Fe coupling, asymmetry appears, and the Fe-Fe distance along the AFM coupling direction is longer than the direction with FM coupling. This magnetic coupling-dependent $\mathrm{Fe}$-Fe distance indicates the presence of spin-lattice coupling. Practically, it means that the magnetic configuration is tunable by introducing external strain along a specific direction. It also implies that the ferromagnetism observed in recent experiments might have been triggered by strain. However, the observation of room-temperature ferromagnetism is still elusive as our calculated Curie temperature for a symmetric ferromagnetic structure is very low, which will be discussed later. It should be mentioned that in addition to the (111) surface, we also studied the (100) and (110) surfaces. Our results show that when the thickness is small, the structure with an AFM configuration always shows lower energy than the FM case. Detailed descriptions and data are given in the Supplemental Material [41].

The calculated band structures are shown in Figs. 1(b) and 1(c). The FM structure has a very small band gap $\sim 50 \mathrm{meV}$ at the $\Gamma$ point between the spin-up valence band maximum with a dominating $\mathrm{S}$ character and spin-down conduction band minimum with a dominating $\mathrm{Fe}$ character. Moreover, both spin channels have their respective band gaps 
( 0.16 and $0.55 \mathrm{eV}$ for the spin-up and spin-down channels). It is interesting to note that electron (hole) doping will give rise to predominantly spin-down (spin-up) conduction. The AFM structure shows a semimetallic behavior with occupied and unoccupied bands meeting at the $\Gamma$ point. It should be noted that a relatively high DOS at the Fermi level for the FM structure compared to the vanishing DOS for the AFM structure reflects the relative stability of the AFM structure. Our calculated MAEs of these structural phases show that the easy axis is along the [100] direction for the AFM structure, and energy differences are around $3.40 \mathrm{meV} / \mathrm{Fe}$ compared to the structure with other magnetization directions ([001], [010], and [110]). For the FM case, due to higher symmetry, the easy axes are along the [100] and [010] directions, and the MAE is $3.27 \mathrm{meV} / \mathrm{Fe}$, which is at the same level as the AFM case.

Calculated total and orbitally resolved exchange parameters of the AFM structure are shown in Fig. 2(a). Each Fe-Fe pair has two exchange parameters due to different couplings along different directions. As seen from Fig. 2(a), the strength of the couplings decreases quickly as the Fe-Fe distance increases. Only the first three nearest neighbors play an essential role, and the most significant contribution comes from the nearest-neighbor couplings. There are six Fe atoms around each $\mathrm{Fe}$ atoms as the first magnetic nearest neighbor; two of them show FM coupling and the other four show AFM coupling. The magnitude of the FM coupling is two times bigger than the AFM one (6.88 and $-2.98 \mathrm{meV}$, respectively). The physical mechanism is understood from orbitally decomposed exchange parameters. As mentioned above, there are four electrons in the $d$ orbital of each $\mathrm{Fe}$ atom, where three of them are located at the $t_{2 g}$ level, and the other one is at the $e_{g}$ level. Figure 2(b) shows a schematic picture of the different exchange interactions. Hopping of the form $e_{g}$-like- $t_{2 g}$-like (hereafter we will call them $t_{2 g}$ and $e_{g}$ to keep the context simple) leads to an exchange coupling that is predominantly FM because of the local Hund's coupling. On the other hand, the $t_{2 g}-t_{2 g}$ hopping is prohibited for FM alignment (shown in the blue box), whereas this is allowed for AFM alignment (shown in the red box). Therefore, $t_{2 g}-t_{2 g}$ hybridization leads to AFM coupling. Another hopping of the form $e_{g}-e_{g}$ changes between AFM and FM as the Fe-Fe distance increases, and by contrast, the strength of this coupling is close to zero and much smaller than the other two and is therefore negligible. For example, the nearest-neighbor ferromagnetic exchange parameter is $6.88 \mathrm{meV}$, of which $e_{g}-t_{2 g}$ and $t_{2 g}-t_{2 g}$ contributions are 7.55 and $-0.63 \mathrm{meV}$, respectively. For the AFM coupling, the contributions come from $e_{g}-t_{2 g}$ and $t_{2 g}-t_{2 g}$ as 1.34 and $-3.49 \mathrm{meV}$, respectively. All of these Fe-Fe exchange interactions are mediated by the hybridization by the $\mathrm{S}-p$ orbitals. The structure-dependent magnetism originates from the dominated form in the coupling. For comparison, we also calculated the exchange parameters and magnetic ordering temperature for the FM structure. Results are shown in Fig. S1. Unlike the AFM structure, the total $J_{i j}$ 's of the FM structure are much smaller due to strong competition between different coupling forms. For the first nearest-neighbor coupling, contributions from $e_{g}-t_{2 g}$ and $t_{2 g}-t_{2 g}$ are 3.73 and $-2.68 \mathrm{meV}$, respectively. As a result, the total exchange parameter is only $0.36 \mathrm{meV}$.
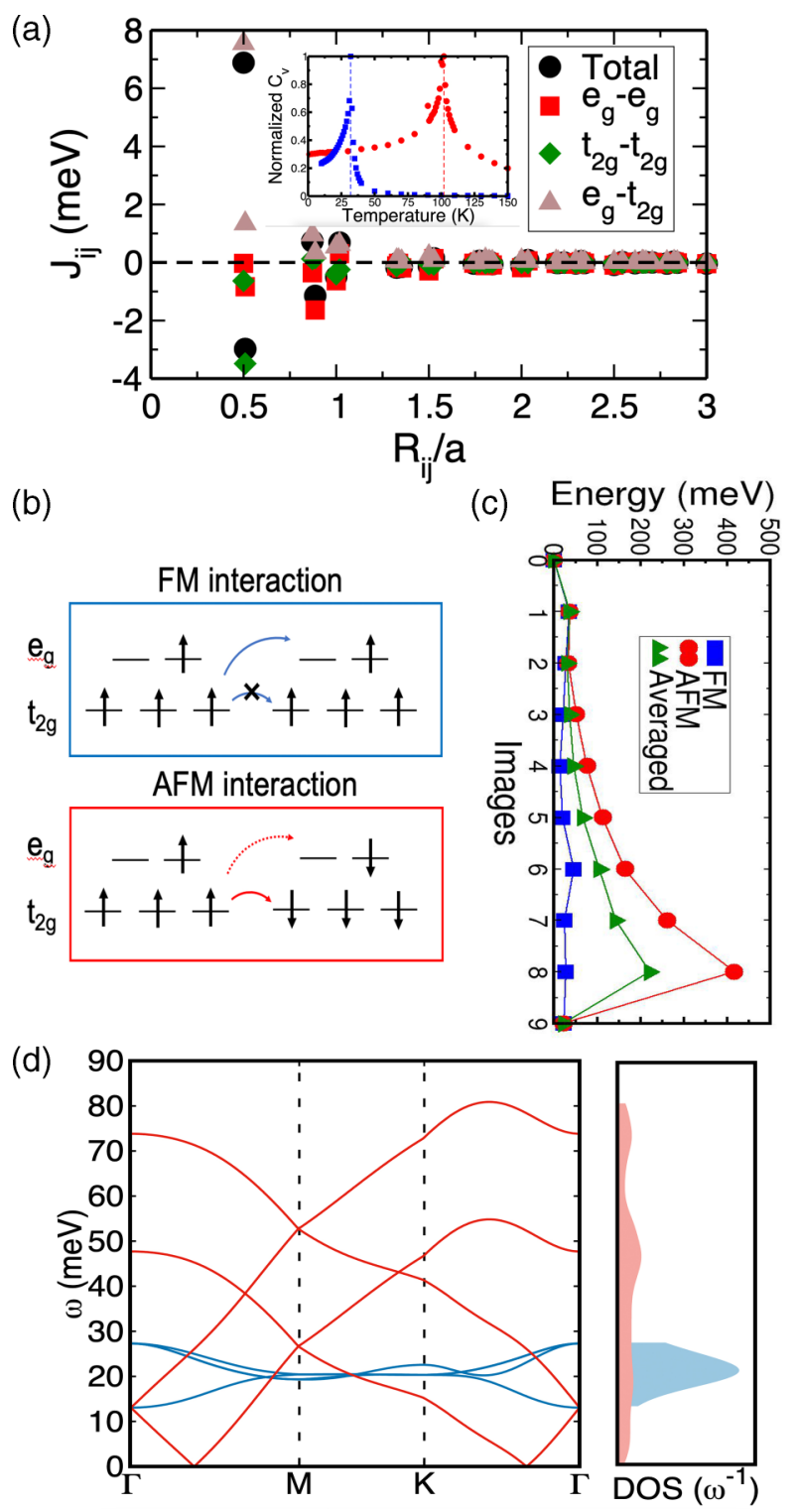

FIG. 2. (a) Calculated total and orbitally resolved exchange parameters of $\mathrm{Fe}-\mathrm{Fe}$ atoms as a function of distance. The positive and negative values indicate ferromagnetic and antiferromagnetic couplings, respectively. The inset shows the normalized specific heat as a function of temperature, calculated from classical Monte Carlo simulations. (b) Schematic picture of the orbital-dependent interactions. (c) Calculated energy barriers from AFM structure to FM structure. For both crystal structures, results for FM and AFM alignments are shown along with the average values. (d) Adiabatic magnon spectra together with magnon DOS for FM (blue) and AFM (red) structures.

In order to estimate the energy involved in the switching between FM and AFM alignments, we have calculate the energy barriers [shown in Fig. 2(c)] by the nudged elastic band method. It is observed that the AFM alignments involve a much large energy barrier compared to the FM alignment. The average values of these two alignments are shown to have a rough estimate, which turns out to be $\sim 200 \mathrm{meV}$. This indicates that it is possible to switch from the ground state AFM to the FM structure by strain, which might have caused ferromagnetism in the recent experiment. 


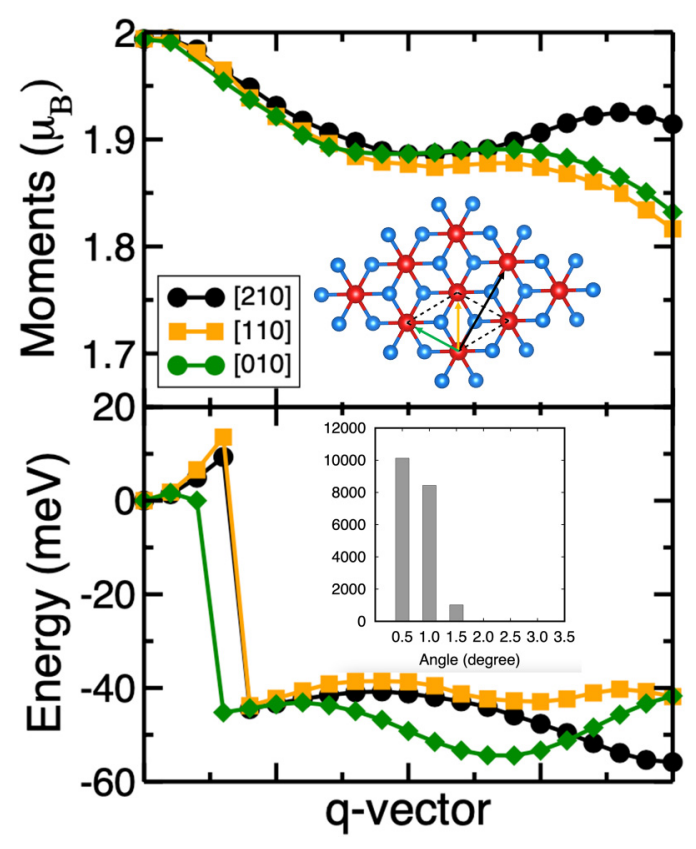

FIG. 3. Calculated magnetic moments (upper panel) and relative spin-spiral energies (lower panel) as a function of the $q$ vector for the AFM structure where the energy at $q=[0,0,0]$ is set to zero. The inset in the upper panel shows a crystal structure with spin-spiral propagation directions [010] (green), [110] (red), and [210] (black), which correspond to the curves in the upper and lower panel, and the unit cell is shown by the dashed lines. The inset in the lower panel shows the histogram of the deviation angles of the magnetic moments relative to the easy axis, obtained from MCS.

Furthermore, we performed classical Monte Carlo simulations (MCS) based on the calculated MAE values and exchange parameters. As shown in the inset in Fig. 2(a), the magnetic ordering temperature of the AFM structure is $102 \mathrm{~K}$, whereas for the FM structure it is $32 \mathrm{~K}$, which reflects the difference in total magnetic exchange parameters between the two.

Finally, based on the calculated exchange parameters and MAE, we calculated adiabatic magnon spectra for both AFM and FM structures as shown in Fig. 2(d). First, there are four magnon branches for both structures, which are dictated by the number of $\mathrm{Fe}$ atoms in the unit cell. Second, the energy gaps at the $\Gamma$ point are around $13 \mathrm{meV}$ for both AFM and FM structures due to the similar MAEs. Third, the dispersion relation around the $\Gamma$ point shows quadratic (linear) dependence for the FM (AFM) structure. At last, as it is shown in the magnon DOS, compared to the AFM structure, the magnon spectrum of the FM structure is more localized.

Based on the generalized Bloch theorem, the spin-spiral total energies can be calculated using the primitive cell. As shown in the inset of the upper panel in Fig. 3, three arrows represent propagation directions of the spin-spiral vector but have been translated into real space. Magnetic moments and relative energies as a function of the propagation vector are shown in the upper and lower panels of Fig. 3. The left and right endpoints represent the FM and AFM states, respectively. For the spin-spiral propagations along the [010] and [110] directions, the AFM state has lower energy than the FM state, which is the same as the result we got from the collinear spin calculations. Remarkably, the magnetic ground state along these two directions is not AFM but spin-spiral states $\left(q_{[010]}=[0.47,-0.23,0], q_{[110]}=[0.23,0.23,0]\right)$. For another propagation direction ([210], black curve), the ground state occurs at the right endpoint - the AFM state $\left[q_{[210]}=\right.$ $(0,0.5,0)]$.

The results for the FM structure are shown in Fig. S2. One difference is obvious if we compare it to the AFM structure: The spin-spiral total energy as a function of $q$ vector in two directions ([010] blue, and [110] red) is exactly the same due to the higher crystal symmetry. The distances of all the first nearest $\mathrm{Fe}-\mathrm{Fe}$ pairs are identical, so the spin-spiral states in these two directions are also identical. These results, on the other hand, demonstrate the strong spin-lattice coupling in $\mathrm{FeS}_{2}$. The magnetic ground state along these two directions is FM, which corresponds to our collinear spin calculations. For the states along another propagation direction ([210]), the AFM configuration has the lowest energy. The stability of the noncollinear magnetic arrangement obtained from our $T=0 \mathrm{~K}$ DFT calculations is also confirmed by the magnetic configurations obtained by Monte Carlo simulations at $T=0.02 \mathrm{~K}$ for the FM and AFM structures. The histogram of the deviation angle of the moments from the easy axis is shown in the inset in Figs. 3 and S2. It is clearly seen that the magnetic moments are deviated from the direction of easy magnetization ([100] direction).

\section{CONCLUSION}

In summary, we performed systematic investigations on the magnetic properties of $\mathrm{FeS}_{2}$ (111) monolayer. We found that the structure with an AFM configuration has lower energy than the structure with the FM configuration with the presence of spin-lattice coupling. The recently reported room-temperature ferromagnetism in experiments may have occurred due to sufficient strain in the samples. We calculated the magnetic exchange parameters and critical temperature for both AFM and FM phases, and due to the different mechanisms of the exchange coupling and the competition between orbital channels, the magnetic ordering temperatures for these two phases are 102 and $32 \mathrm{~K}$, respectively. Our calculated spin-spiral energies using the generalized Bloch theorem and also supported by the results from Monte Carlo simulations indicate that the magnetic ground states of the ground state structural phase are not perfectly collinear. The complex magnetism in the $\mathrm{FeS}_{2}$ (111) monolayer, tunable by strain, may open up interesting applications in spin-driven devices.

Note added. Recently, we became aware of an experimental paper [29] in which the authors revised the results as they pointed out that they observed a ferromagnetic phase, but it only appears at low temperature, which is consistent with our theoretical studies.

\section{ACKNOWLEDGMENTS}

B.S. acknowledges financial support from the project Grant (2016-05366) and the Swedish Research Links programme Grant (2017-05447) from Swedish Research Council, and acknowledges Carl Tryggers Stiftelse for providing Grant 
No. CTS 20:378. D.W. thanks the China scholarship council for financial support (No. 201706210084). B.S. and D.W. gratefully acknowledge supercomputing time alloca- tion by the Swedish National Infrastructure for Computing (Project No. SNIC2020-3-26) and PRACE DECI-15 project DYNAMAT.
[1] L. Kou, Y. Ma, Z. Sun, T. Heine, and C. Chen, Two-dimensional topological insulators: progress and prospects, J. Phys. Chem. Lett. 8, 1905 (2017).

[2] Y. O. Kvashnin, A. Bergman, A. I. Lichtenstein, and M. I. Katsnelson, Relativistic exchange interactions in $\mathrm{CrX} 3$ (X = Cl, Br, I) monolayers, Phys. Rev. B 102, 115162 (2020).

[3] J. Li, Y. Li, S. Du, Z. Wang, B.-L. Gu, S.-C. Zhang, K. He, W. Duan, and $\mathrm{Y} . \mathrm{Xu}$, Intrinsic magnetic topological insulators in van der Waals layered $\mathrm{MnBi}_{2} \mathrm{Te}_{4}$-family materials, Sci. Adv. 5, eaaw5685 (2019).

[4] J. Zhang, X. Shen, Y. Wang, C. Ji, Y. Zhou, J. Wang, F. Huang, and X. Lu, Design of Two-Dimensional Multiferroics with Direct Polarization-Magnetization Coupling, Phys. Rev. Lett. 125, 017601 (2020).

[5] X. Feng, J. Liu, X. Ma, and M. Zhao, Ferroelectricity and multiferroicity in two-dimensional $\mathrm{Sc}_{2} \mathrm{P}_{2} \mathrm{Se}_{6}$ and $\mathrm{ScCrP}_{2} \mathrm{Se}_{6}$ monolayers, Phys. Chem. Chem. Phys. 22, 7489 (2020).

[6] C. Gong, E. M. Kim, Y. Wang, G. Lee, and X. Zhang, Multiferroicity in atomic van der Waals heterostructures, Nat. Commun. 10, 2657 (2019).

[7] S.-H. Zhang and B.-G. Liu, A controllable robust multiferroic $\mathrm{GaTeCl}$ monolayer with colossal 2D ferroelectricity and desirable multifunctionality, Nanoscale 10, 5990 (2018).

[8] C. Gong and X. Zhang, Two-dimensional magnetic crystals and emergent heterostructure devices, Science 363, eaav4450 (2019).

[9] B. Huang, G. Clark, E. Navarro-Moratalla, D. R. Klein, R. Cheng, K. L. Seyler, D. Zhong, E. Schmidgall, M. A. McGuire, D. H. Cobden, W. Yao, D. Xiao, P. Jarillo-Herrero, and X. Xu, Layer-dependent ferromagnetism in a van der Waals crystal down to the monolayer limit, Nature (London) 546, 270 (2017).

[10] C. Gong, L. Li, Z. Li, H. Ji, A. Stern, Y. Xia, T. Cao, W. Bao, C. Wang, Y. Wang, Z. Q. Qiu, R. J. Cava, S. G. Louie, J. Xia, and X. Zhang, Discovery of intrinsic ferromagnetism in twodimensional van der Waals crystals, Nature (London) 546, 265 (2017).

[11] Z. Fei, B. Huang, P. Malinowski, W. Wang, T. Song, J. Sanchez, W. Yao, D. Xiao, X. Zhu, A. F. May, W. Wu, D. H. Cobden, J.$\mathrm{H}$. Chu, and $\mathrm{X} . \mathrm{Xu}$, Two-dimensional itinerant ferromagnetism in atomically thin $\mathrm{Fe}_{3} \mathrm{GeTe}_{2}$, Nat. Mater. 17, 778 (2018).

[12] H. Li, Y.-K. Xu, K. Lai, and W.-B. Zhang, The enhanced ferromagnetism of single-layer $\mathrm{CrX}_{3}(\mathrm{X}=\mathrm{Br}$ and $\mathrm{I})$ via van der Waals engineering, Phys. Chem. Chem. Phys. 21, 11949 (2019).

[13] M. Grönke, B. Buschbeck, P. Schmidt, M. Valldor, S. Oswald, Q. Hao, A. Lubk, D. Wolf, U. Steiner, B. Büchner, and S. Hampel, Chromium trihalides $\mathrm{CrX}_{3}(\mathrm{X}=\mathrm{Cl}, \mathrm{Br}, \mathrm{I})$ : Direct deposition of micro- and nanosheets on substrates by chemical vapor transport, Adv. Mater. Interfaces 6, 1901410 (2019).

[14] D. J. O'Hara, T. Zhu, A. H. Trout, A. S. Ahmed, Yunqiu, Luo, C. H. Lee, M. R. Brenner, S. Rajan, J. A. Gupta, D. W. McComb, and R. K. Kawakami, Room Temperature Intrinsic Ferromagnetism in Epitaxial Manganese Selenide Films in the Monolayer Limit, Nano Lett. 18, 3125 (2018).
[15] D. Lançon, R. A. Ewings, T. Guidi, F. Formisano, and A. R. Wildes, Magnetic exchange parameters and anisotropy of the quasi-two-dimensional antiferromagnet $\mathrm{NiPS}_{3}$, Phys. Rev. B 98, 134414 (2018).

[16] R. Kumar, R. N. Jenjeti, M. P. Austeria, and S. Sampath, Bulk and few-layer $\mathrm{MnPS}_{3}$ : A new candidate for field effect transistors and UV photodetectors, J. Mater. Chem. C 7, 324 (2019).

[17] A. R. Wildes, V. Simonet, E. Ressouche, R. Ballou, and G. J. McIntyre, The magnetic properties and structure of the quasitwo-dimensional antiferromagnet $\mathrm{CoPS}_{3}$, J. Phys.: Condens. Matter 29, 455801 (2017).

[18] X. Sun, W. Li, X. Wang, Q. Sui, T. Zhang, Z. Wang, L. Liu, D. Li, S. Feng, S. Zhong, H. Wang, V. Bouchiat, M. N. Regueiro, N. Rougemaille, J. Coraux, A. Purbawati, A. Hadj-Azzem, Z. Wang, B. Dong, X. Wu, T. Yang, G. Yu, B. Wang, Z. Han, X. Han, and Z. Zhang, Room temperature ferromagnetism in ultrathin van der Waals crystals of $1 \mathrm{~T}-\mathrm{CrTe}_{2}$, Nano Res. 13, 3358 (2020).

[19] Y. Wen, Z. Liu, Y. Zhang, C. Xia, B. Zhai, X. Zhang, G. Zhai, C. Shen, P. He, R. Cheng, L. Yin, Y. Yao, M. G. Sendeku, Z. Wang, X. Ye, C. Liu, C. Jiang, C. Shan, Y. Long, and J. He, Tunable room-temperature ferromagnetism in two-dimensional $\mathrm{Cr}_{2} \mathrm{Te}_{3}$, Nano Lett. 20, 3130 (2020).

[20] A. Ennaoui, S. Fiechter, H. Goslowsky, and H. Tributsch, Photoactive synthetic polycrystalline pyrite $\left(\mathrm{FeS}_{2}\right)$, J. Electrochem. Soc. 132, 1579 (1985).

[21] A. Ennaoui and H. Tributsch, Iron sulphide solar cells, Iron sulphide solar cells, Sol. Cells 13, 197 (1984).

[22] D. Banjara, Y. Malozovsky, L. Franklin, and D. Bagayoko, First-principles studies of electronic, transport and bulk properties of pyrite $\mathrm{FeS}_{2}$, AIP Adv. 8, 025212 (2018).

[23] A. Hung, J. Muscat, I. Yarovsky, and S. P. Russo, Densityfunctional theory studies of pyrite $\mathrm{FeS}_{2}(100)$ and (110) surfaces, Surf. Sci. 513, 511 (2002).

[24] A. Hung, J. Muscat, I. Yarovsky, and S. P. Russo, Densityfunctional theory studies of pyrite FeS2 (111) and (210) surfaces, Surf. Sci. 520, 111 (2002).

[25] D. R. Alfonso, Computational investigation of $\mathrm{FeS}_{2}$ surfaces and prediction of effects of sulfur environment on stabilities, J. Phys. Chem. C 114, 8971 (2010).

[26] F. Herbert, A. Krishnamoorthy, K. V. Vliet, and B. Yildiz, Quantification of electronic band gap and surface states on $\mathrm{FeS}_{2}$ (100), Surf. Sci. 618, 53 (2013).

[27] H. Zhang, Y.-M. Dai, and L.-M. Liu, Novel monolayer pyrite $\mathrm{FeS}_{2}$ with atomic-thickness for magnetic devices, Comput. Mater. Sci. 101, 255 (2015).

[28] A. B. Puthirath, A. P. Balan, E. F. Oliveira, L. Deng, R. Dahal, F. C. R. Hernandez, G. Gao, N. Chakingal, L. M. Sassi, T. Prasankumar, G. Costin, R. Vajtai, C. W. Chu, D. S. Galvao, R. R. Nair, and P. M. Ajayan, Exfoliated Pyrite $\left(\mathrm{FeS}_{2}\right)$ - a nonvan der Waals 2D Ferromagnet, arXiv:2010.03113v1 (2020).

[29] A. B. Puthirath, A. P. Balan, E. F. Oliveira, V. Sreepal, F. C. R. Hernandez, G. Gao, N. Chakingal, L. M. Sassi, P. Thibeorchews, G. Costin, R. Vajtai, D. S. Galvao, R. R. Nair, 
and P. M. Ajayan, Apparent ferromagnetism in exfoliated ultrathin pyrite sheets, J. Phys. Chem. C 125, 18927 (2021).

[30] W. Kohn and L. J. Sham, Self-consistent equations including exchange and correlation effects, Phys. Rev. 140, A1133 (1965).

[31] P. E. Blöchl, Projector augmented-wave method, Phys. Rev. B 50, 17953 (1994).

[32] G. Kresse and D. Joubert, From ultrasoft pseudopotentials to the projector augmented-wave method, Phys. Rev. B 59, 1758 (1999).

[33] G. Kresse and J. Furthmüller, Efficiency of ab-initio total energy calculations for metals and semiconductors using a plane-wave basis set, Efficiency of ab-initio total energy calculations for metals and semiconductors using a plane-wave basis set, Comput. Mater. Sci. 6, 15 (1996).

[34] J. M. Wills, M. Alouani, P. Andersson, A. Delin, O. Eriksson, and O. Grechnyev, Full-Potential Electronic Structure Method: Energy and Force Calculations with Density Functional and Dynamical Mean Field Theory (Springer, Berlin, 2010), Vol. 167.

[35] A. I. Liechtenstein, M. I. Katsnelson, V. P. Antropov, and V. A. Gubanov, Local spin density functional approach to the theory of exchange interactions in ferromagnetic metals and alloys, J. Magn. Magn. Mater. 67, 65 (1987).

[36] J. P. Perdew, K. Burke, and M. Ernzerhof, Generalized Gradient Approximation Made Simple, Generalized Gradient Approximation Made Simple, Phys. Rev. Lett. 77, 3865 (1996).
[37] S. L. Dudarev, G. A. Botton, S. Y. Savrasov, C. J. Humphreys, and A. P. Sutton, Electron-energy-loss spectra and the structural stability of nickel oxide: An LSDA $+U$ study, Electron-energy-loss spectra and the structural stability of nickel oxide: An LSDA+U study, Phys. Rev. B 57, 1505 (1998).

[38] A. P. Balan, S. Radhakrishnan, C. F. Woellner, S. K. Sinha, L. Deng, C. d. 1. Reyes, B. M. Rao, M. Paulose, R. Neupane, A. Apte, V. Kochat, R. Vajtai, A. R. Harutyunyan, C.-W. Chu, G. Costin, D. S. Galvao, A. A. Martí, P. A. van Aken, O. K. Varghese, C. S. Tiwary, A. M. M. R. Iyer, and P. M. Ajayan, Exfoliation of a non-van der Waals material from iron ore hematite, Nat. Nanotechnol. 13, 602 (2018).

[39] O. Eriksson, A. Bergman, L. Bergqvist, and J. Hellsvik, Atomistic Spin Dynamics: Foundations and Applications (Oxford University Press, Oxford, UK, 2017).

[40] G. Henkelman, B. P. Uberuaga, and H. Jónsson, A climbing image nudged elastic band method for finding saddle points and minimum energy paths, A climbing image nudged elastic band method for finding saddle points and minimum energy paths, J. Chem. Phys. 113, 9901 (2000).

[41] See Supplemental Material at http://link.aps.org/supplemental/ 10.1103/PhysRevB.104.245410 for total and orbitally resolved exchange parameters for a ferromagnetic structure, spin-spiral total energies and corresponding magnetic moments as a function of spin-spiral vector for the ferromagnetic structure, and optimized geometries, magnetic moments, and energetics of (100) and (110) surfaces. 